To be sure that your preprints are promptly included in the HIGH ENERGY PHYSICS INDEX,

send them to the following address (if possible by air mail) :

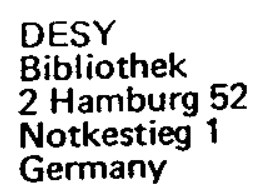


Hadron Production by Virtual Photons in the Quark

\section{Fragmentation Regio}

b y

J.M. Scarr, C.K. Chen", J. Knowles, D. Martin, I.O. Skillicorn, and K. Smith, University of Glasgow, Glasgow

P. Joos, A. Ladage, H. Meyer, ${ }^{+}$and G. Wolf

Deutsches Elektronen-Synchrotron DESY, Hanburg

G. Drews, J. Knobloch ${ }^{++}$, H. Nage1, E. Rabe, W. -D. Schlatter, H. Spitzer, and $K$. Wacker ${ }^{+}$

II. Institut ftir Experimentalphysik der Universität Hamburg, Hamburg

\section{Abstract}

We have measured the inclusive electroproduction of positive and negative hadrons in the quark fragmentation region using the streamer chamber at DESY. Data are presented in terms of the variable $z_{p}=p / v$ in the kinematic region $1.8<\mathrm{W}<2.8 \mathrm{GeV}$ and $0.3<\mathrm{Q}^{2}<1.4 \mathrm{GeV}^{2}$. The positive hadron distributions contain a strong proton component. After subtraction of the proton component and elastic rho events, the distribution $1 / \sigma_{\text {tot }}$ $\left(\mathrm{d} \sigma / \mathrm{d} \mathrm{p}_{\mathrm{p}}\right)$ for positive and negative hadrons agrees well with the corresponding distribution from $\mathrm{e}^{+} \mathrm{e}^{-}$annihilation (DORIS data). This behaviour supports the validity of the quark parton model at surprisingly low $Q^{2}$ and $W$.

* Now at Argonne National Laboratory, Argonne, I1l., USA.

+ Now at Bergische Universität, Wuppertal, Germany

++ Now at CERN, Geneva, Switzeriand '
1. Introduction

The observation in 1968 of approximate scaling of the total inelastic ep scattering cross section for $Q^{2}>1 \mathrm{GeV}^{2}$ and $W>2 \mathrm{GeV}$ initiated the interpretation of inelastic processes in terms of quark parton models. In the last few years an impressive amount of data has been accumulated from $e^{+} e^{-}$, ep and hp interactions which can be understood in terms of quark parton models $\mathrm{s}^{1-3}$. However, most of these data were obtained at considerably higher energies than the region $W=2-3 \mathrm{GeV}$ where the onset of scaling has been observed.

We have measured the electroproduction of positive and negative hadrons in the transition region where scaling starts, i.e. $1.8<\mathrm{W}<2.8 \mathrm{GeV}$ and $0.3<Q^{2}<1.4 \mathrm{GeV}^{2}$. We here report on a study of our inclusive data with the object of finding whether the scaling properties expected from the quark parton model apply at these low energies.

We consider only the current fragmentation region, where the quark parton model leads to specific predictions ${ }^{2}$ :

1) There should be an excess of positive over negative pions.

2) The sum of the $\pi^{+}$and $\pi^{-}$cross sections is twice the $\pi^{\circ}$ cross section.

3) The normalized distributions for $\left(h^{+}+h^{-}\right)$-production from ep and $\mathrm{e}^{+} \mathrm{e}^{-}$interactions should be similar.

In order to check the above predictions we eliminate from our data backgrounds not due to quark parton fragmentation. We subtract both elastic rho production $\gamma_{v} p \rightarrow e^{o} p$, and the substantial number of forward protons which are not produced by current fragmentation. Having applied these cuts our data agree with the quark parton model predictions.

In the following we discuss the experimental procedure in section 2, and the choice of the variable $z_{p}$ used for analyzing the data in section 3 . In section 4 we present the $z_{p}$ distributions and the $\mathrm{h}^{+} / \mathrm{h}^{-}$ratio in the current fragmentation region. Finally, the data are compared to predictions of the quark parton model in section 5 . 


\section{Experimental Procedure}

Positive and negative hadrons from inelastic ep scattering at $7.2 \mathrm{GeV}$ beam energy have been detected in a $1 \mathrm{~m}$ streamer chamber at DESY. The data sample and analysis procedure is the same as that presented in ref. 4. This paper is based on 11300 reconstructed events in the kinematic region $1.8<\mathrm{W}<2.8$ GeV and $0.3<Q^{2}<1.4 \mathrm{GeV}^{2}$. The data have been corrected for 1 imited acceptance of the electron detection system and for particle losses in the streamer chamber. The $Q^{2}$ and $W$ dependence of the topological cross sections has been radiatively corrected. We have not, however, corrected the $z_{p}$ distributions for smearing in $z_{p}$ due to measuring errors and radiation.

We analyze the electroproduction of hadrons in terms of the proces

$$
\gamma_{\mathrm{v}} \mathrm{p} \rightarrow \mathrm{hX}
$$

assuming that the interaction is produced by a 'beam' of virtual photons. The connection between the electron and the virtual photon scattering cross sections can be found in ref. 5 .

\section{The Choice of the Variable $z$}

In the quark-parton model the virtual photon is assumed to be absorbed by a single quark, or antiquark, which, behaving as a free particle, is ejected from the target proton and subsequently fragments into the hadrons of the current fragmentation region. The breakup of the quarks is described by the fragmentation functions ${ }^{2} D_{q}^{h}(z)$, which represent the probability of a quark, q, giving a hadron, h, carrying a fraction $z$ of the quark energy. For electroproduction $z$ is given in invariant form by

$$
z=\frac{(P \cdot h)}{(P \cdot q)}
$$

where $q, P$, and $h=(E, \vec{p})$ are the 4-momenta of the virtual photon, target proton, and final hadron respectively. In terms of laboratory frame variables

$$
z=\mathrm{E} / \mathrm{v}
$$

where $E$ is the hadron energy, and $\nu$ the photon energy.
In practice we use the common alternative

$$
\mathrm{z}_{\mathrm{p}}=\mathrm{p} / \mathrm{v}
$$

which does not require particle identification. At our energies $z_{p}$ differs from $z$ by less than $3 \%$ for pions with $z>0.2$.

In the current fragmentation region, $z_{p}$ is closely related to the variable $\mathrm{x}_{\mathrm{f}}=\mathrm{p}_{\mathrm{tl}}^{*} / \mathrm{p}_{\max }^{*} \cdot z_{\mathrm{p}} \approx x_{\mathrm{F}}$ if $\varepsilon / \nu \approx \mathrm{p}_{11} / \nu$, that is, for high energies and positive $x_{F}$ values not close to $x_{F}=0$. Under these circumstances the invariant structure function can be written

$$
E \frac{d^{3} q}{d^{3} \vec{p}}=\frac{x_{F}}{\pi} \frac{d^{2} \sigma}{d x_{F} d p_{\perp}^{2}} \approx \frac{z_{p}}{\pi} \frac{d^{2} \sigma}{d z_{p} d p_{\perp}{ }^{2}} \quad f\left(z_{p}, p_{\perp}{ }^{2}\right)
$$

In most figures, however, we use the normalized distribution ${ }^{*}$

$$
F\left(z_{p}\right)=\frac{1}{\sigma_{\text {tot }}} \frac{d \sigma}{d z_{p}} \text {, where }
$$

$F\left(z_{p}\right) d z_{p}$ is the number of particles produced per event in the interval $z_{p} \rightarrow z_{p}+d z_{p}$. The inclusive $\pi^{-}$distributions in terms of the variable $x_{F}$ and $p_{\perp}$ have been presented for our data in ref. 4.

\section{The $z_{p}$ Distributions for positive and negative Hadrons}

First, we describe the procedure used to subtract the elastically produced rhos. Roughly $7 \%$ of the total inelastic cross section in our kinematic range proceeds via quasi-elastic $\rho$ production $\gamma_{v} p \rightarrow \rho^{0}$. This process is known to be dominantly diffractive ${ }^{5}$. It yields pions which are mainly in the forward region $\left(z_{p}>0.3\right)$. Since we are interested in the quark fragnentation properties and not in the diffractive contributions we subtract the $\rho$ contribution by omitting a11 $\gamma_{\mathrm{v}} \mathrm{p}_{\mathrm{p}} \pi^{+} \pi^{-}$events with $\mathrm{M}_{\pi \pi}<1 \mathrm{GeV}$.

Fig. I shows the normalized distribution

$$
\frac{z_{p}}{\pi \sigma \sigma_{\text {tot }}} \frac{d \sigma}{d z_{p}}=\frac{z_{p}}{\pi} F\left(z_{p}\right)
$$

for positive and negative hadron production in the interaval $2.2<W<2.8 \mathrm{GeV}$

* For comparison

with other particle beams we normalize $f$ to the total inelastic ep scattering cross section, $\sigma_{\text {tot }}$, and also integrate over $p_{\perp}$. 
and $0.3<Q^{2}<1.4 \mathrm{GeV}^{2}$ (fu11 points) ${ }^{*}$. The crosses show the same data after the rho subtraction. Note that the latter cross section has been normalized to $\sigma_{\text {tot }}-\sigma_{\rho}$. The $\rho$ subtraction has 1 ittle effect on the normalized $h^{+}$ distribution ${ }^{\circ}$, whereas the $\mathrm{h}^{-}$distribution is reduced in the current fragmentation region. The small excess of positive hadrons with $z_{p}>1$ has to be attributed to fast protons for which values of $p>v$ are kinematically allowed. We now discuss the $\mathrm{h}^{+} / \mathrm{h}^{-}$ratio and its $\mathrm{Q}^{2}$ dependence in the current fragmentation region. Fig. 2 shows the normalized distribution

$$
F\left(z_{p}\right)=\frac{1}{\sigma_{\text {tot }}} \frac{d \sigma}{d z_{p}}
$$

for $\mathrm{h}^{+}$and $\mathrm{h}^{-}$production in the $W$ interval $1.8-2.2 \mathrm{GeV}$ for two $Q^{2}$ intervals. Clearly there is a large excess of positive over negative hadrons. The data sets at $\left\langle Q^{2}\right\rangle=0.4 \mathrm{GeV}^{2}$ and $\left\langle Q^{2}\right\rangle=0.9 \mathrm{GeV}^{2}$ agree within errors. Fig. 3 shows the corresponding distributions for $2.2<\mathrm{W}<2.8 \mathrm{GeV}$. Again positive hadrons dominate over negative ones. We observe no $Q^{2}$ dependence. These trends are more clearly illustrated in fig. 4, where the integral of $F\left(z_{p}\right)$ from $z_{\mathrm{p}}=0.4$ to $z_{\mathrm{p}}=0.9$ is shown. The $\mathrm{h}^{+} / \mathrm{h}^{-}$ratio is about 10 at $\langle\mathrm{W}\rangle=2 \mathrm{GeV}$ and decreases to 4.65 at $\langle W\rangle=2.5 \mathrm{GeV}$. In view of the $Q^{2}$ independence of the data we present in the following the data averaged over the interval $0.3<Q^{2}$ $1.4 \mathrm{Gev}^{2}$.

In our data, the low walue makes it kinematically possible for proton from target fragmentation to attain large $z_{p}$ values, thus contaminating the current fragmentation distributions. Since current fragmentation is expected to produce only a small fraction of protons, the proton background should be removed from our data before comparison is made with other processes, such as $\mathrm{e}^{+} \mathrm{e}^{-} \rightarrow$ hadrons. Furthermore, the electroproduction of protons is strongly

*) The total inelastic $\gamma_{\mathrm{v}} \mathrm{p}$ cross section was determined by normalizing our data to the total inelastic cross section measured in a single arm experiment ${ }^{6}$. For consistency with our previous paper ${ }^{4}$ we have excluded events with visible strange particle decay (1\% of the events) both from the inclusive data and the above total cross section.

**) This implies that positive hadrons (consisting of $\pi^{+}$and protons) from tho events have the same $z_{p}$ distribution as those from non-rho events.
W dependent ${ }^{7-10}$ and the proton component largely explains the strong $W$ dependence of $F\left(z_{p}\right)$ for positive hadrons.

We therefore remove the proton contribution from our distributions using the data of Ackermann et al. ${ }^{9}$ which cover most of our kinematic region ${ }^{*}$. Ackermann et al. have parametrized their data, which is independent of $Q^{2}$, as a function of $W, p_{1}^{2}$ and $x_{F}=p_{11}^{*} / p_{\max }^{*}$ (eq. 5.1 of ref, 10). We have transformed their cross sections in small intervals of $p_{\perp}^{2}$ from $x_{F}$ to $z_{P}$ and have obtained the corresponding $F\left(z_{p}\right)$ distribution by integration over $p_{\perp}^{2}$. We estimate that the resulting $F\left(z_{p}\right)$ is accurate to within $20 \%$.

Fig. 5 and Table 1 show the $F\left(z_{p}\right)$ distribution for positive hadrons before (full circles) and after the proton subtraction (open circles).

The crosses are from negative hadrons ${ }^{* *}$. The $\mathrm{h}^{+} / \mathrm{h}^{-}$ratio is now reduced to values of 2 in agreement with measurements at $3.9<\mathrm{W}<5.6 \mathrm{GeV}$ where the proton contribution is sma11 ${ }^{11 * * *}$.

\section{Test of Parton Model Predictions}

In this section we compare our data to the reactions $\gamma_{v^{p}} \rightarrow \pi^{0} x$ and $e^{*} e^{-}+h^{ \pm} x$ with the object of testing quark parton model predictions in the energy. interval $2.2<\mathrm{W}<2.8 \mathrm{GeV}$.

The parton mode1 is specified by two sets of functions, the quark distribution functions $q(x)(q=u, \bar{u}, d, \bar{d}, s, \bar{s})$ and the quark fragmentation functions $D_{q}^{h}(z)$. $\mathrm{x}=\mathrm{Q}^{2} / 2 \mathrm{MV}$ is the fraction of the proton momentum carried by the quark.

*) The data of ref. 9 cover the region $0.08<Q^{2}<0.64 \mathrm{GeV}^{2}, 1.9<W<2.8$ Gev, $0<x_{F}<1$ and $0<p_{\perp}^{2}<0.29 \mathrm{GeV}^{2}$. The measured $p_{\perp}^{2}$ distribution can be fitted by $\exp \left(-2.5 p_{\perp}^{2}\right)$ for $x_{F}>0.4$. We assume the same $p_{\perp}^{2}$ dependence up to $\mathrm{p}_{\perp}^{2}=1 \mathrm{GeV}^{2}$

**) The peak in the $\mathrm{h}^{-}$-distribution near $\mathrm{z}_{\mathrm{p}}=0.85$ is due to the reaction $\gamma_{v} p \rightarrow \pi^{-} \Delta^{++}$.

***) We estimate that about $15-20 \%$ of the remaining positive hadrons are $\mathrm{K}^{+}$mesons (for $2.2<\mathrm{W}<2.8 \mathrm{GeV}$ ). This estimate is based on the $\mathrm{K}^{+}$inclusive data of ref, 12 and on our $\mathrm{h}^{+}$data. The $\mathrm{K}^{-} / \mathrm{h}^{-}$ratio is expected to be smaller than $0.17,11$. 
$q(x) d x$ then gives the probability of finding a quark of type $q$ in an interval between $x$ and $x+d x \cdot D_{q}^{h}(z) d s$ gives the probability of a hadron $h$ fragmenting from a quark of type $q$ in the inverval $z \rightarrow z+d z$.

For electroproduction the quark-parton model of ref. 2 predicts

$\frac{1}{\sigma_{\text {tot }}} \frac{d \sigma}{d z_{p}}\left(\gamma, p \rightarrow \pi^{-} X\right)$

$$
=\frac{\frac{4}{g}\left(u(x) D_{u}^{\pi^{-}}+\bar{u}(x) D_{u}^{\pi^{+}}\right)+\frac{1}{g}\left(d(x) D_{u}^{\pi^{+}}+\bar{d}(x) D_{u}^{\pi^{-}}+s(x) D_{u}^{\pi^{-}}+\bar{s}(x) D_{u}^{\pi^{+}}\right)}{\frac{4}{9}(u(x)+\bar{u}(x))+\frac{1}{g}(d(x)+\bar{d}(x)+s(x)+\bar{s}(x))}
$$

$\frac{1}{\sigma_{\text {tot }}} \frac{d \sigma}{d z_{p}}\left(\gamma_{r} p \rightarrow \pi^{+} X\right)$

$$
=\frac{\frac{4}{9}\left(u(x) D_{u}^{\pi^{+}}+\bar{u}(x) D_{u}^{\pi^{-}}\right)+\frac{1}{9}\left(d(x) D_{u}^{\pi^{-}}+\bar{d}(x) D_{u}^{\pi^{+}}+s(x) D_{u}^{\pi^{-}}+\bar{s}(x) D_{u}^{\pi^{-}}\right)(1 b)}{\frac{4}{9}(u(x)+\bar{u}(x))+\frac{1}{9}(d(x)+\bar{d}(x)+s(x)+\bar{s}(x))}
$$

Equations 1 lead to the prediction ${ }^{2}$

$$
\begin{aligned}
\frac{1}{\sigma_{\text {tot }}}\left[\frac{d \sigma}{d z_{p}}\left(\gamma_{v} p \rightarrow \pi^{-} x\right)\right. & \left.+\frac{d \sigma}{d z_{p}}\left(\gamma_{v} p \rightarrow \pi^{+} x\right)\right] \approx D_{u^{\pi^{+}}}+D_{u}^{\pi^{*}} \\
& =2 D_{u}^{\pi^{\circ}}=\frac{2}{\sigma_{\text {tot }}} \frac{d \sigma}{d z_{p}}\left(\gamma_{v} p \rightarrow \pi^{\circ} x\right)
\end{aligned}
$$

Qualitatively, we expect an excess of $\pi^{+}$over $\pi^{-}$, since $\pi^{+}$are formed more easily than $\pi^{-}$from the dominant $u$ quarks; the u quarks dominate over the $d$ quarks by virtue of their greater probability of occurrence in the proton and their larger coupling to the virtual photon.

The distribution for $\mathrm{e}^{+} \mathrm{e}^{-}$annihilation (neg1ecting the small contributions from $s$ and $\bar{s}$ quarks) can be related to the electroproduction distribution by

$$
\frac{1}{\sigma_{t+i t}} \frac{d \sigma}{d z_{p}}\left(\gamma_{v} p \rightarrow h^{ \pm} X\right) \approx \frac{1}{2} \frac{1}{\sigma_{t o t}} \frac{d \sigma}{d z_{p}}\left(e^{+} e^{-} \rightarrow h^{ \pm} x\right)
$$

The factor $1 / 2$ in $\mathrm{Eq}$. (3) arises from the fact that two quarks are fragmenting in $\mathrm{e}^{+} \mathrm{e}^{-}$annibilation.

The distributions of $F\left(z_{p}\right)=\frac{1}{\sigma} \frac{d \sigma}{d z_{p}}$ have been calculated for $\pi^{+}$and $\pi^{-}$ electroproduction using eqs. lat and $b$ with the parametrization of $D(z)$ and $u(x), \bar{u}(x) \ldots$, given in ref. 2. The resulting curves are shown in fig. 5 and are in good agreement with the data.
We now compare our results with other data to test the relations of eqs. 2 and 3. In figure 6 we compare our $\mathrm{h}^{-}$distribution (which consists of $\pi^{-}$mesons together with $a \leqslant 10 \% \mathrm{~K}^{-}$contamination) with recent data of Berger et al. ${ }^{13}$ on $\pi^{\circ}$ production (full circles) at $W=2.8 \mathrm{GeV}$ and $Q^{2}=0.45 \mathrm{GeV}^{2}$. Generally the $\pi^{-}$data are lower than the $\pi^{\circ}$ data. When we average our $\pi^{-}$and $\pi^{+}$data (using the open points in fig. 5) we find that eq. (2) is well satisfied at our energies (open circles). The same agreement was observed in ref. 13 using data at higher $w$.

Fig. 7 and table 1 show our $\mathrm{h}^{+}+\mathrm{h}^{-}$data (with protons subtracted). The curve gives the average of $\frac{1}{2} \frac{1}{\sigma \sigma_{t o t}} \frac{d \sigma}{d z}\left(e^{t} e^{-} \rightarrow h^{ \pm} x\right)$ measured recently by the PLUTO group at $\mathrm{W}=3.6 \mathrm{GeV} 14$. The $\mathrm{PLUT}$ data agree with our results and also with the corresponding data from DASP15*. We emphasize that the good agreement of our electroproduction data with the DORIS data is only obtained after subtracting the contribution of forward scattered protons and elastic rho production. This has not been done in previous comparisons of electro- and muonproduction data with the SPEAR results $2,17,18$.

The comparison in Fig. 7 is made for the sum of $\pi$ and $\mathrm{K}$ mesons (neglecting the relatively small proton contribution in the $\mathrm{e}^{+} \mathrm{e}^{-}$data). We note that although there is general agreement there may be some differences in detail. For example there is a tendency for more kaons to be produced in $e^{+} e^{-}$annihilation ${ }^{15}$ than in electroproduction ${ }^{1}$, however, this seems to be compensated by a higher $\pi$ yield in electroproduction for $z_{p}>0.6$ (see figs. 4 and 5 of ref. 13).

\section{Surmary and Conclusions}

We have measured the $F\left(z_{p}\right)=\frac{1}{\sigma \sigma_{t} t} \frac{d \sigma}{d z_{p}}$ distribution for electroproduced $\pi^{+}$and $\pi^{-}$mesons in the kinematic region $0.3<Q^{2}<1.4 \mathrm{GeV}^{2}$ and $2.2<W<2.8$. The contribution from diffractive $\rho^{\circ}$ production, $\gamma_{v} p \rightarrow \rho^{0} p$, has been subtracted. Furthermore, the background from fast protons, which is significant at our energies, has also been subtracted. The resulting distributions have relative kaon contributions of $\mathrm{K}^{-} / \pi^{-} 60.1$ and $\mathrm{K}^{+} / \pi^{+} \leqslant 0.2$.

*) In contrast the spectra from SPEAR $^{16}$ are high by factors of $2-3$ for $z_{p}>0.6$. 
Our resulting $F\left(z_{\mathrm{p}}\right)$ distributions have been compared with expectations obtained using the quark fragmentation functions, $D_{q}(z)$, of Feynman and Field ${ }^{2}$ and with equivalent distributions obtained from $\gamma_{v^{p}} \rightarrow \pi^{0} x^{13}$ and $e^{+} e^{-}+h^{ \pm} x^{14}$. The agreement is found to be good.

\section{Acknowledgements}

We thank C. Benz, I. Bloodworth, D. Hoffmann, W. Kraus, B. Naroska, D. Notz, W.J. Podolsky, C. Sander, P. Stein, and S. Yellin for their help in the early stages of this experiment. We thank also $\mathrm{N}$. Gollmer, E. Hell, v. Heynen, A. Huber, H. Klinkmüller, G. Kraft, H.H. Sabath, S.W. Sass, K. Westpha1, and K.H. Wroblewski for their technical assistance, We acknowledge the excellent performance of the Synchrotron crew, the Hallendienst and the Kaltetechnik. The cooperation by Mr. Kuhlmann and the Rechenzentrum has been invaluable. We thank our scanning and measuring staffs for their careful work.

The Glasgow members of the collaboration thank the DESY Directorate for their warm hospitality. The work at Hamburg has been supported by the Bundesministerium für Forschung und Technologie, the work at Glasgow by the Science Research Council.

\section{References}

1. J.T. Dakin and G.J. Feldman, Phys. Rev. D8 (1973) 2862, S.J. Brodsky and J.F. Gunion, Proceedings of the VII International Colloquium on Multiparticle Reactions, Tutzing 1976, p. 369; ed. by 3. Benecke et al., MPI für Physik, Miinchen.

2. R. D.Field and R.P. Feynman, Phys. Rev. D15 (1977) 2590, R.P.Feynman, R.D.Field and G.C. Fox, Nucl. Phys, B128 (1977) 1.

3. A. Seiden, Phys. Letters 683 (1977) 157 and SLAC-PUB-1962 (1977), Submitted to Nucl. Phys.

4. C. K.Chen, J. Knowles, D. Martin, J.M. Scarr, I.O. Skillicorn, P. Joos, A. Ladage, H. Meyer, P. Stein, G. Wolf, S. Yellin, C. Benz, G. Drews, D. Hoffmann, J. Knobloch, W. Kraus, H. Nagel, E. Rabe, C. Sander, W.-D. Schlatter, H. Spitzer and K, Wacker, DESY $77 / 69$ (1977), submitted to Nuc1. Phys. B

5. P. Joos et a1., Nuc1. Phys. B113 (1976) 53.

6. S. Stein et a1., Phys. Rev. D12 (1975) 1884.

7. J. C. Alder et al,, Nuc1. Phys. B46 (1972) 415.

8. C.J. Bebeck et a1., Phys. Rev. Letters 32 (1974) 27.

9. H. Ackermann et a1,, NucI. Phys. B120 (1977) 365.

10. H. Ackermann, Thesis, Universität Marburg, 1976 (unpublished).

11. J.F. Martin et al., Phys, Letters 65B (1976) 483.

12. H. Ackermann et al., Inclusive Spectra of Electroproduced $\mathrm{x}^{+}$ Mesons, DESY $77 / 49$ (1977).

13. Ch. Berger, R. Bühring, G. Dick, R. Grigul1, W. Wagner, H. Ackermann, T. Azemoon, W. Gabriel, H.D. Mertiens, H.D. Reich, F. Janata and D.Schmidt, DESY $77 / 47$ (1977).

14. PLUTO group; Talk of G. Knies at the 1977 International Symposium on Lepton and Photon Interactions at High Energies, Hamburg, 1977.

15. R, Brandelik et a1., Phys, Letters 67B (1977) 358 
Table 1: $F\left(z_{\mathrm{p}}\right)=\frac{1}{\sigma_{\text {tot }}} \frac{\mathrm{do}}{\mathrm{dz}}$ for $0.3<\mathrm{Q}^{2}<1.4 \mathrm{GeV}^{2}$ and $2.2<\mathrm{W}<2.8 \mathrm{GeV}$

Elastic tho events. have been excluded as described in section 4

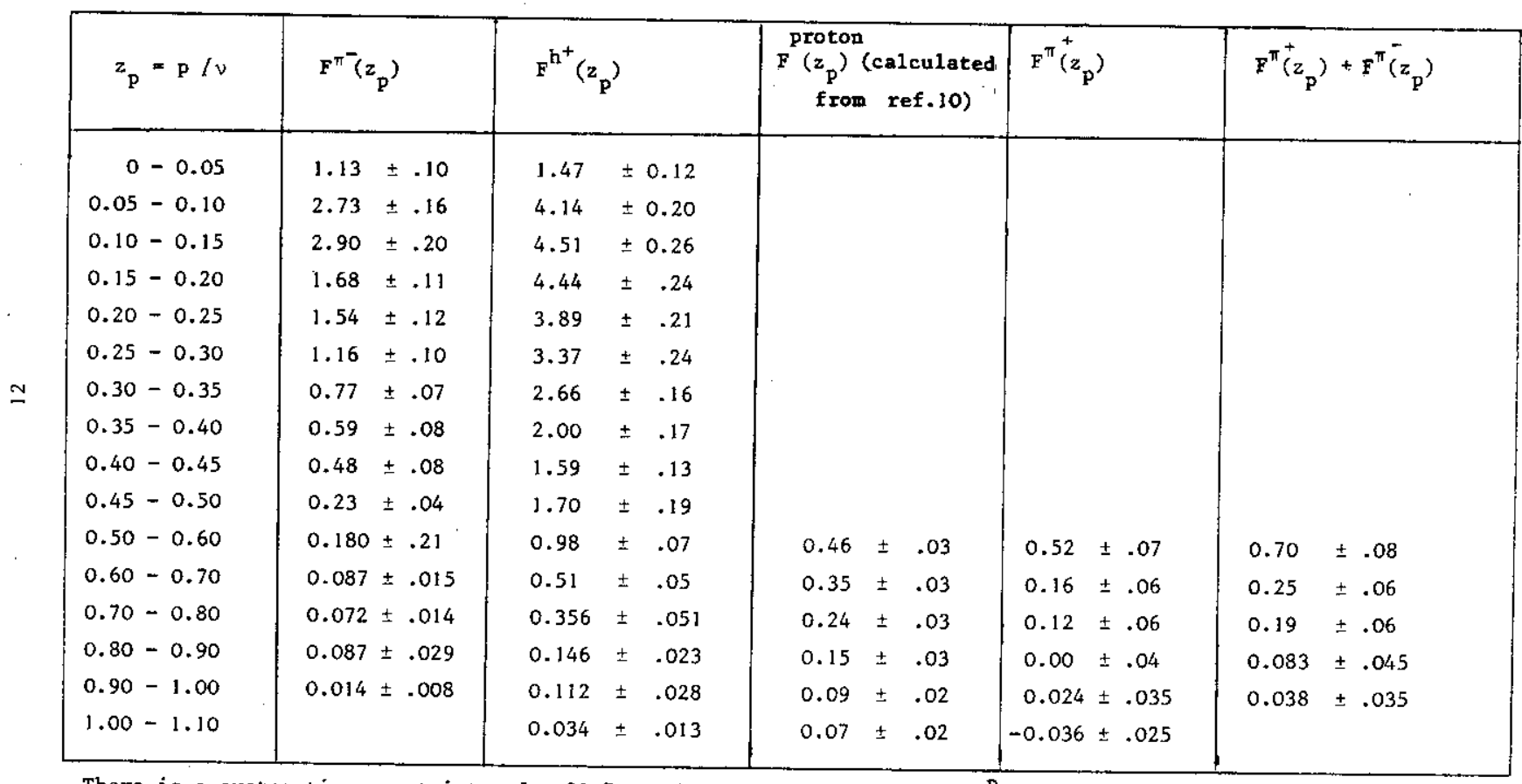

There is a systematic uncertainty of $\sim 20 \%$ on the calculated values of $F^{P}(z)$ not included in the table. The $F^{\pi^{-}}$ distributions include a $\leqslant 10 \% \mathrm{~K}^{-}$contamination. The $\mathrm{F}^{\pi^{+}}$distributions include a $\leqslant 20 \% \mathrm{~K}^{+}$contamination. 
Figure Captions

Fig. 1: Inclusive hadron distributions for reactions $\gamma_{\mathrm{y}} \mathrm{p} \rightarrow \mathrm{h}^{\mathrm{t}} \mathrm{x}$ and $\gamma_{\mathrm{v}} \mathrm{p} \rightarrow \mathrm{h}^{-} \mathrm{X}$ for $2.2<\mathrm{W}<2.8 \mathrm{GeV}$ and $0.3<\mathrm{Q}^{2}<1.4 \mathrm{GeV}^{2}$. Full points: elastic rho included. Crosses: Elastic rho events were excluded by omitting all $\gamma_{\mathrm{v}} \mathrm{p}^{+} \mathrm{p} \pi^{+} \pi^{-}$events with $M_{\pi \pi}<1 \mathrm{GeV}$. The latter distribution has been normalized to $\sigma_{\text {tot }}-\sigma_{\rho}$.

Fig. 2: Inclusive hadron distributions for $1.8<\mathrm{W}<2.2 \mathrm{GeV}$ and two $Q^{2}$ intervals (elastic rho excluded as in Fig. 1).

$\omega$ is the scaling variable $\omega=1 / x=2 \mathrm{M} v / \mathrm{Q}^{2}$.

Fig. 3: Inclusive hadron distributions for $2.2<\mathrm{W}<2.8 \mathrm{GeV}$ (elastic rho exc1uded)

Fig. 4: Average value of $\frac{1}{\sigma_{\text {tot }}} \frac{d \sigma}{d z}$ for the interval $0.4<z_{p}<0.9$ as a function of $Q^{2}$ for positive (squares) and negative hadrons (circles). The elastic rho contribution is excluded.
a) $1.8<\mathrm{W}<2.2 \mathrm{GeV}$,
b) $2.2<W<2.8 \mathrm{GeV}$.

Fig. 5: Inclusive distribution of positive (full points) and negative hadrons (crosses) for $2.2<\mathrm{W}<2.8 \mathrm{GeV}$ and $0.3<\mathrm{Q}^{2}<1.4 \mathrm{GeV}^{2}$ (elastic rho excluded). The open points are from positive hadrons after a subtraction of protons. The subtraction is based on proton data of ref. 9. The curves are predictions for $Y_{v} p \rightarrow \pi^{-} X$ (full curve) and $\gamma_{v^{p} \rightarrow \pi}{ }^{+} X$ (dashed curve) using the formulae of ref. 2 .

Fig. 6: Inclusive distributions for the reactions $\gamma_{\mathrm{v}} \rightarrow \mathrm{h}^{-} \mathrm{X}$ (crosses) from this experiment (elastic rho excluded) compared with $\gamma_{v} p \rightarrow \pi^{\circ} x$ (full points from ref. 13). The open points show $1 / 2\left(\mathrm{~F}^{+}(\mathrm{z})+\mathrm{F}^{-}(\mathrm{z})\right)$ from this experiment.

Fig. 7: Inclusive distributions of the sum of positive and negative hadrons (full points). The open points were obtained after a subtraction of forward emitted protons. The curve gives the average $h^{+}+h^{-}$ distribution from $\mathrm{e}^{+} \mathrm{e}^{-} \rightarrow \mathrm{h}^{ \pm} \mathrm{X}$ (ref, 14) at an electron beam energy of $1.8 \mathrm{GeV}$.

The spectra of ref. 14 are not corrected for radiative effects. We therefore normalized these data to a total $\mathrm{e}^{+} \mathrm{e}^{-}$cross section which is also uncorrected for radiation $\left(\sigma_{\text {tot }}=18 \mathrm{nb}\right.$; private communication from A. Bäcker).

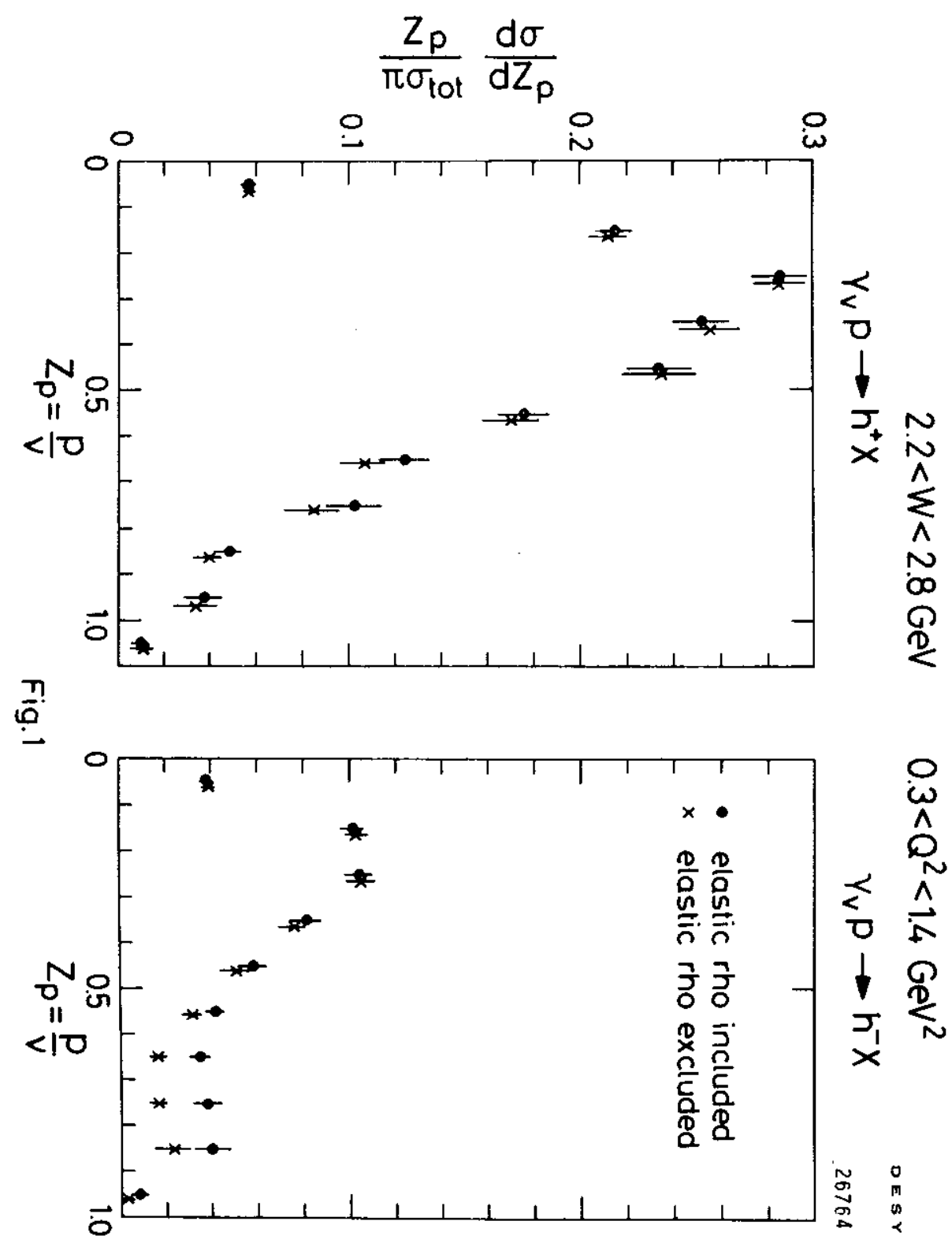



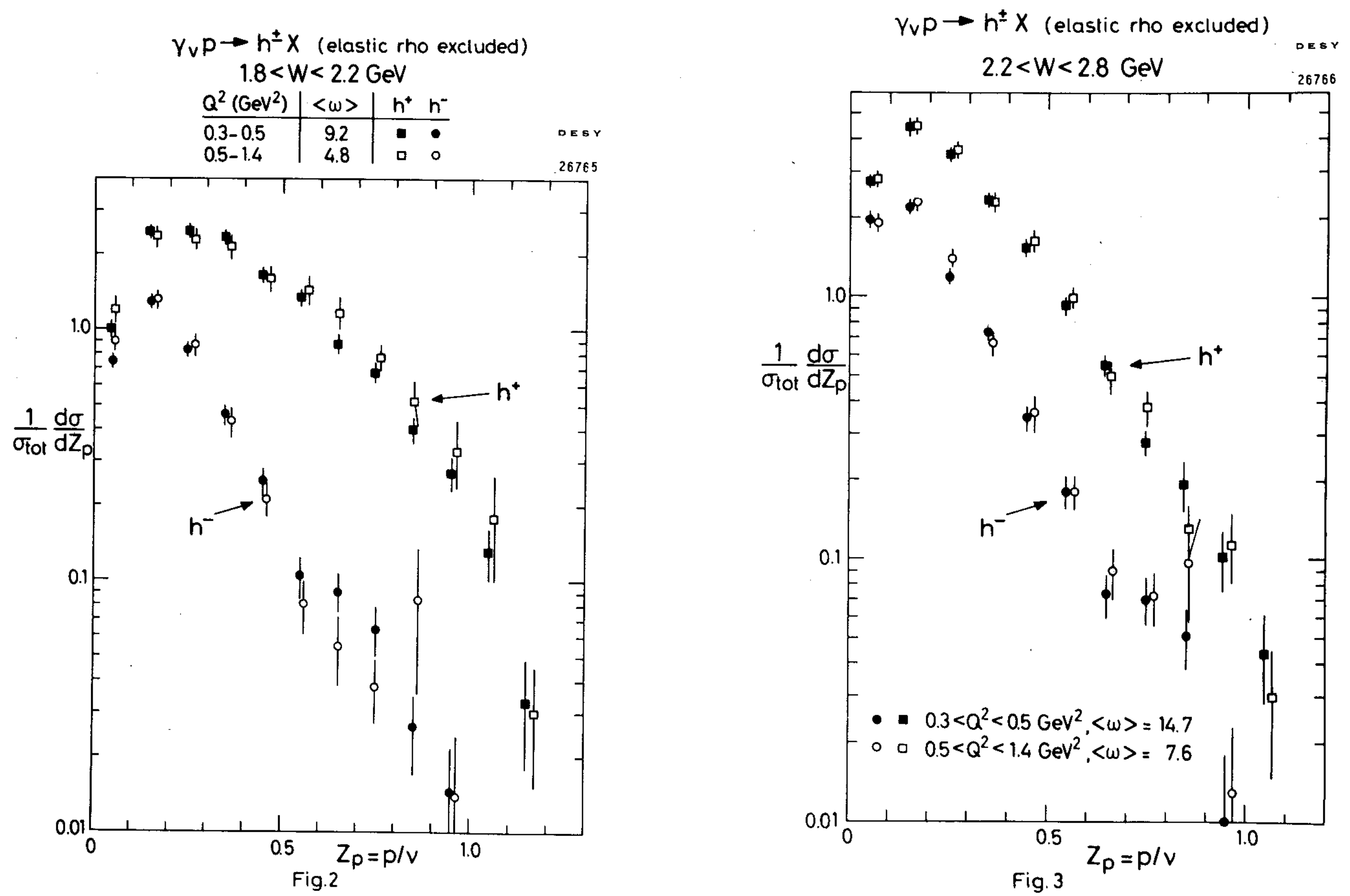

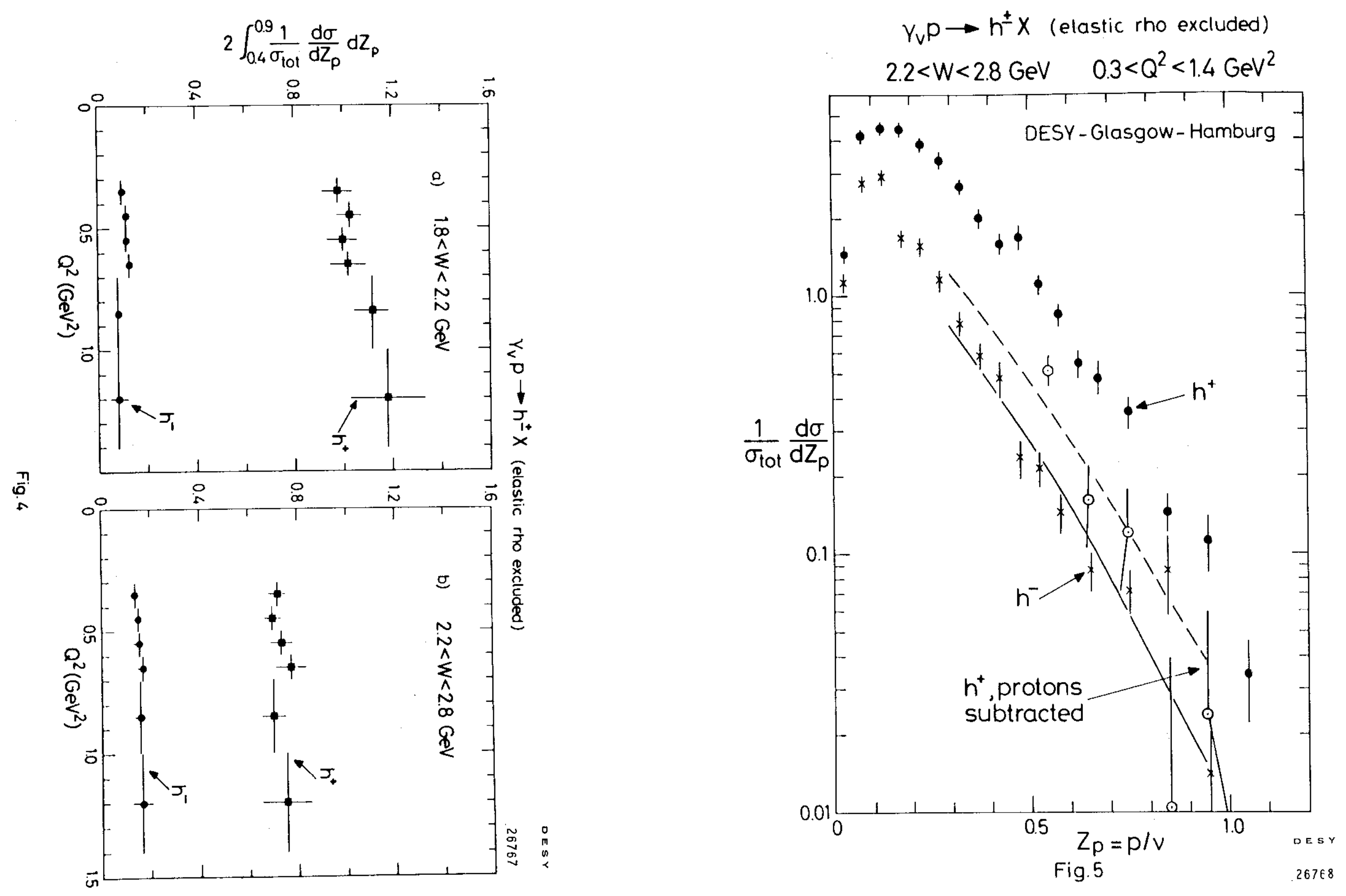

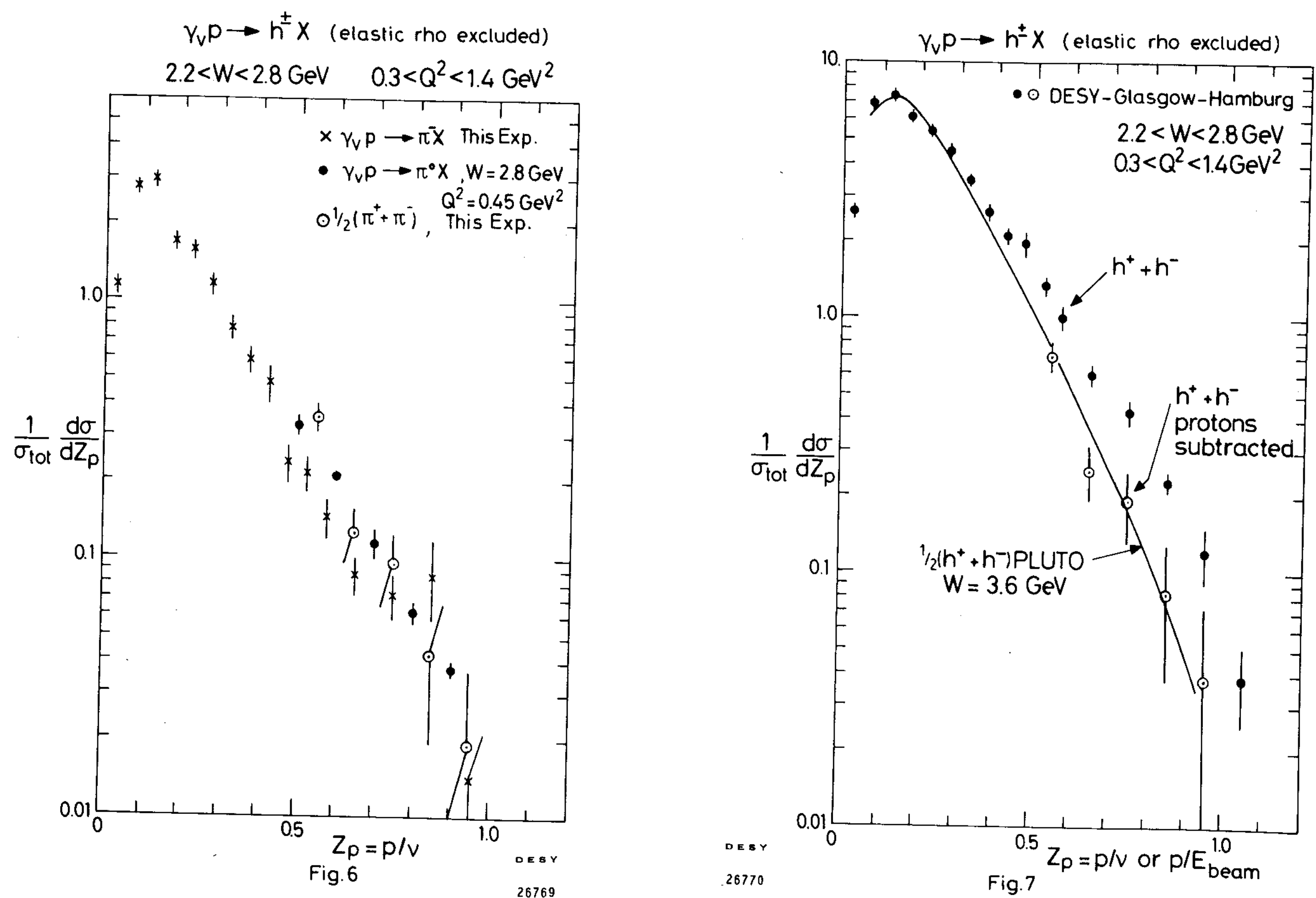